Copyright 2008 Society of Photo-Optical Instrumentation Engineers.

This paper was published in Proceedings of SPIE, vol. 6915, Medical Imaging 2008: Computer Aided Diagnosis and is made available as an electronic reprint with permission of SPIE. One print or electronic copy may be made for personal use only. Systematic or multiple reproduction, distribution to multiple locations via electronic or other means, duplication of any material in this paper for a fee or for commercial purposes, or modification of the content of the paper are prohibited. 


\title{
Human Airway Measurement from CT Images
}

\author{
Jaesung Lee ${ }^{a}$, Anthony P. Reeves ${ }^{a}$, Sergei Fotin ${ }^{a}$, \\ Tatiyana Apanasovich ${ }^{b}$, and David Yankelevitz ${ }^{c}$ \\ ${ }^{a}$ School of Electrical and Computer Engineering, Cornell University, Ithaca, NY; \\ ${ }^{b}$ School of Operations Research and Information Engineering, Cornell University, Ithaca, NY; \\ ${ }^{c}$ Department of Radiology, Weill Cornell Medical College, New York, NY
}

\begin{abstract}
A wide range of pulmonary diseases, including common ones such as COPD, affect the airways. If the dimensions of airway can be measured with high confidence, the clinicians will be able to better diagnose diseases as well as monitor progression and response to treatment. In this paper, we introduce a method to assess the airway dimensions from CT scans, including the airway segments that are not oriented axially. First, the airway lumen is segmented and skeletonized, and subsequently each airway segment is identified. We then represent each airway segment using a segment-centric generalized cylinder model and assess airway lumen diameter (LD) and wall thickness (WT) for each segment by determining inner and outer wall boundaries. The method was evaluated on 14 healthy patients from a Weill Cornell database who had two scans within a 2 month interval. The corresponding airway segments were located in two scans and measured using the automated method. The total number of segments identified in both scans was 131. When 131 segments were considered altogether, the average absolute change over two scans was $0.31 \mathrm{~mm}$ for LD and $0.12 \mathrm{~mm}$ for WT, with $95 \%$ limits of agreement of $[-0.85,0.83]$ for LD and $[-0.32,0.26]$ for WT. The results were also analyzed on per-patient basis, and the average absolute change was $0.19 \mathrm{~mm}$ for $\mathrm{LD}$ and $0.05 \mathrm{~mm}$ for WT. $95 \%$ limits of agreement for per-patient changes were $[-0.57,0.47]$ for LD and $[-0.16,0.10]$ for WT.
\end{abstract}

Keywords: Quantitative image analysis, X-ray CT, diagnosis, airway

\section{INTRODUCTION}

Airway dimension is an important indicator for diagnosis of certain class of pulmonary diseases. Currently, whole-lung CT images are the main source for such information. Manual analysis of CT scans is hard due to the amount of visual data to process and complicated by the properties of human airway tree. Anatomically, individual segments of a human airway tree have tubular structure, where low intensity lumen is surrounded by high intensity wall and attached structures. Image of lumen for a particular segment on a CT scan is not perfectly cylindrical: cross-sections are not perfectly circular and centerline may be bent to a certain extent. Even though the position of large airways may be determined by human anatomy, smaller ones can have arbitrary orientations.

There have been previous attempts to automatically measure airway dimensions. The algorithms proposed in the past ${ }^{1,2}$ can only handle the airway segments that are oriented axially. Renhardt et al. ${ }^{1}$ proposed a method to estimate the inner and outer radius of the airway. They used a maximum-likelihoold method using the profile of a ray crossing the airway wall. Their method, however, was designed for $2 \mathrm{D}$ slices and limited to axially oriented airway segments. Nakano et al. ${ }^{2}$ used a semi-automated airway measurement algorithm. They measured the area of the airway wall area as well as the lumen and wall perimeters. Their method required a seed point from which rays are projected outward. Full width at half maximum principle was used with manual editing of rays that go beyond the airway wall area.

The previous studies ${ }^{1,3}$ have used phantom data for validating their methods. Tschirren et al. ${ }^{3}$ proposed an airway tree segmentation algorithm that avoids leaks by using adaptive region of interest. They also performed lumen diameter measurement by determining the inner wall boundary with dynamic programming. The lumen

Further author information: (Send correspondence to Jaesung Lee) Jaesung Lee: E-mail: j1569@cornell.edu, Telephone: 16072550963 


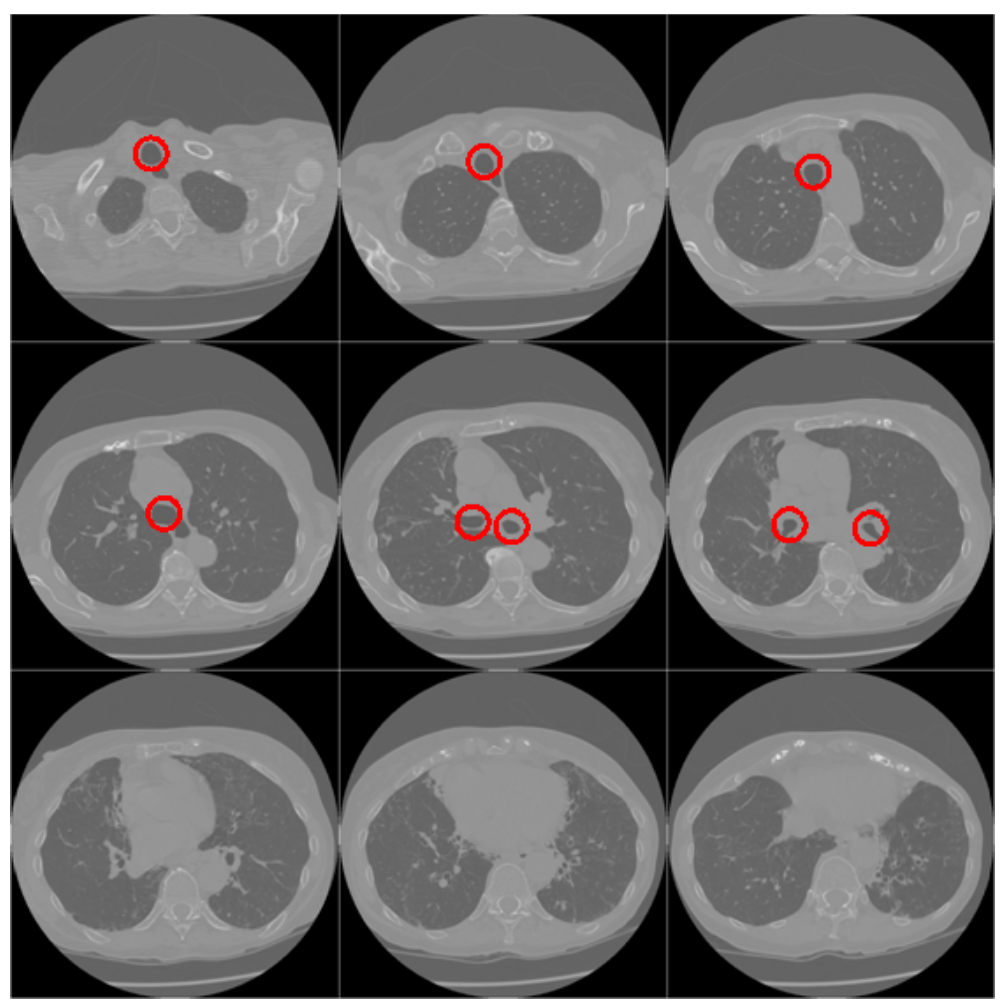

Figure 1. Airways in chest CT slices. Trachea and main bronchi are marked with circles. Each main bronchus splits further into smaller bronchial tubes.

diameter measurement was validated with 6 phantoms with maximum measurement error of $0.31 \mathrm{~mm}$ for low-dose scans.

Many studies ${ }^{1,2,4}$ were limited to evaluating the airway on a manually selected single CT slices, instead of considering whole airway segment spanning multiple CT images. Berger et al. ${ }^{4}$ attempted to separate the high-intensity wall area from the lumen area and lung parenchyma using a Laplacian of Gaussian algorithm. Manual editing of the wall area was required to completely separate two regions. The lumen and wall area were measured once the regions have been separated.

Skeletonization of tree structure and branch point identification are important steps in measuring airway dimensions. Palágyi et al. ${ }^{5}$ introduced an algorithm to extract the centerlines of tubular tree structure and identify branch points. Their algorithm was developed to account for imaging artifacts present in volumetric medical images.

The purpose of this research was to develop a robust method for assessing human airway dimensions from CT scans. The method should be able to deal with the arbitrarily oriented airway segments and should be automated with no need for manual intervention. Further, the measurements must have high precision for monitoring the changes in airway segments across multiple scans. In this paper, we introduce a method for representation and analysis of airways that can handle arbitrarily oriented airway segments and considers the complete length of the segment.

\section{METHODOLOGY}

The computer algorithm to measure airway diameter and wall thickness consists of three stages. First, the airway segments are identified from a whole-lung CT scan. Second, the spatial region for each airway segment is mapped to a segment-centric generalized cylinder model. Finally, the diameter and wall thickness for the segment are estimated from the cylindrical model. 


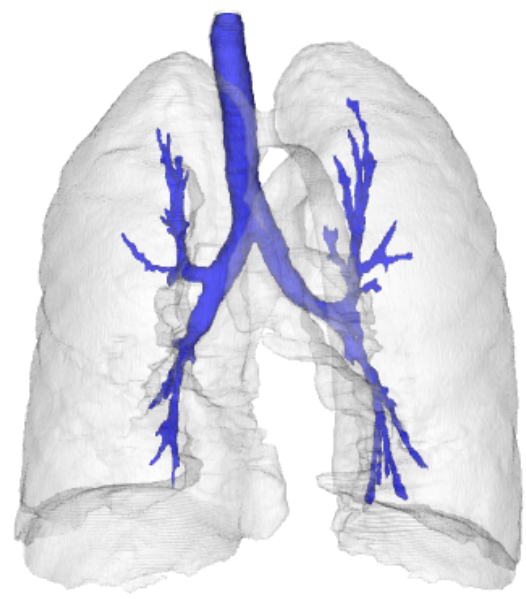

(a)

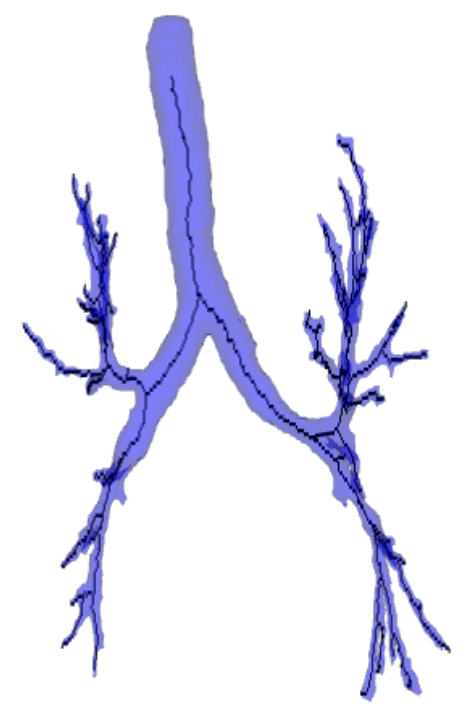

(b)

Figure 2. Segmentation and skeletonization of airway tree. (a) Segmented airway tree and lung outline. (b) Skeletonization of the segmented airway tree. Centerline is shown with the transparent airway tree.

\subsection{Segmentation}

The first step is to segment the airway lumen from the whole-lung CT slices and identify the airway segments from the whole airway tree. Figure 1 shows several axial images of the chest CT, with trachea and main bronchi marked by circles. The trachea (top-most airway) splits into two main bronchi, and each bronchi subsequently splits into smaller airways.

The airway lumen is segmented from the whole-lung CT scan using a 3D region growing algorithm ${ }^{6}$ with automatic seed point detection. Once the airway lumen is segmented, a 3D thinning algorithm is applied to obtain the centerline of the airway tree. Our implementation of thinning uses the simple point criteria proposed by Bertrand et al. ${ }^{7}$ The thinned airway tree is post-processed to obtain smoother centerline. ${ }^{5}$ Figure 2 gives a coronal visualization of the airway tree segmentation and the resulting skeleton for the whole lung. The tree structure skeleton is then traversed to identify and label the sub-skeleton segments starting from the top-most point in the skeleton (the trachea).

\subsection{Cylinder Modeling}

Each identified airway segment is represented by a cylindrical model. The cylinder model (Figure 3a) is defined with $I$ layers and $J$ triangular meshes per layer. The parameter $I$ determines the vertical resolution of the segment along the z-axis of the cylinder, and the parameter $J$ determines the radial sampling resolution within a layer.

In our model, the centerline of each airway segment is defined as a three-dimensional parametric curve $X(t)$ in $\Re^{3}$, as shown in Equation (1).

$$
X(t)=\langle x(t) y(t) z(t)\rangle, \quad t \in[0,1]
$$

where $X(0)$ corresponds to the start point and $X(1)$ to the end point of the segment's centerline. The curve is approximated by fitting three cubic polynomials over the $\mathrm{x}, \mathrm{y}$, and $\mathrm{z}$ coordinates of the points that belong to the airway segment of interest, as shown in Equation (2).

$$
\hat{X}(t)=\sum_{m=0}^{3} A_{m} t^{m}, \quad A_{m}=\left\langle a_{x m} a_{y m} a_{z m}\right\rangle,
$$




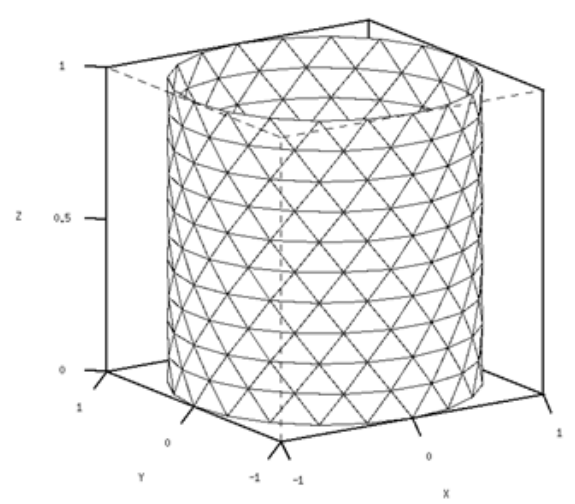

(a)
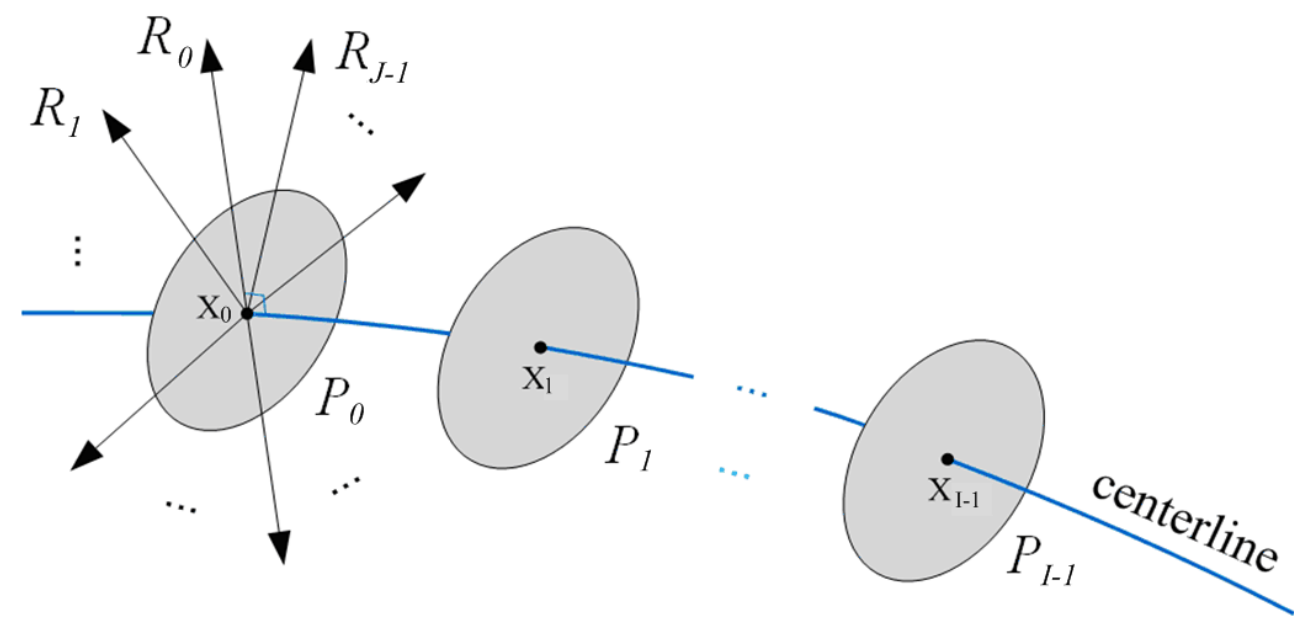

(b)

Figure 3. Airway segment modeling. (a) Cylinder model. (b) Intensity mapping. The rays $R_{j}$ are cast outward from the center points $X_{i}$ toward the centers of the triangles in the cylinder model to sample the intensities.

where $\hat{X}(t)$ is the approximated centerline, and $a_{x}, a_{y}$, and $a_{z}$ are the polynomial coefficients. Approximation of the centerline is done using least squares fit with cubic polynomials, separately for $\mathrm{x}, \mathrm{y}$, and $\mathrm{z}$ coordinates. We have found that the cubic polynomial is adequate to approximate the curved trajectory of a single airway segment.

For each segment, once the centerline $\hat{X}(t)$ and the cylinder model has been defined, a mapping of $\hat{X}(t)$ to the z-axis of the cylinder model is performed. Along the centerline $\hat{X}(t)$, the center points $X_{i}$ are considered, where $i=0,1, \ldots, I-1$. $X_{i}$ corresponds to $\hat{X}\left(t_{i}\right)$, where $t_{i}$ is the $\mathrm{z}$ value of corresponding layer in the cylinder model. For each center point $X_{i}$, tangent vector $T_{i}$ is defined. Then, a sampling plane $P_{i}$ is defined so that the plane is perpendicular to the tangent vector $T_{i}$. For our polynomially approximated model, $T_{i}$ is calculated as shown in Equation (3).

$$
T_{i}=T\left(t_{i}\right)=\left.\frac{d \hat{X}(t)}{d t}\right|_{t=t_{i}}=\sum_{m=1}^{3} A_{m} m t_{i}^{m-1} .
$$

From $X_{i}$, the rays $R_{j}$ are cast outward on the sampling plane $P_{i}$, where $j=0,1, \ldots, J-1$. Figure $3 \mathrm{~b}$ illustrates how the rays are cast from the center points.

The direction of first ray within each sampling plane should be preserved to prevent twisting planes within the model (i.e. spatial orientation of the sampled model in the neighboring planes must be preserved). In order to do this, we define two vectors $R_{x}(i)$ and $R_{y}(i)$ that are perpendicular to each other and lie on $P_{i}$. These 


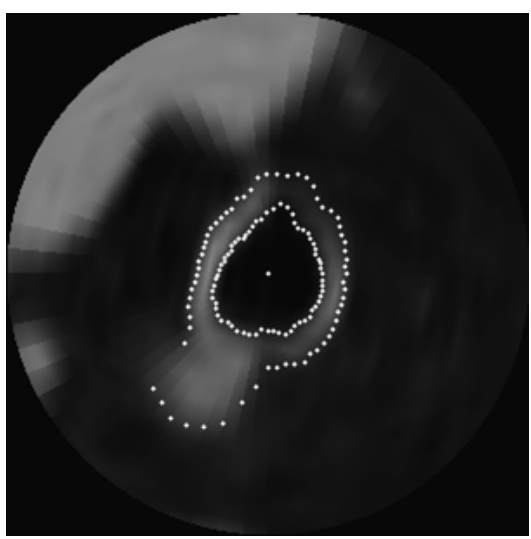

(a)

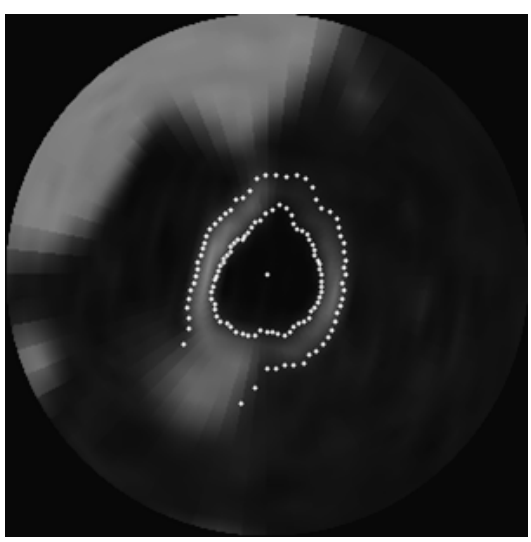

(b)

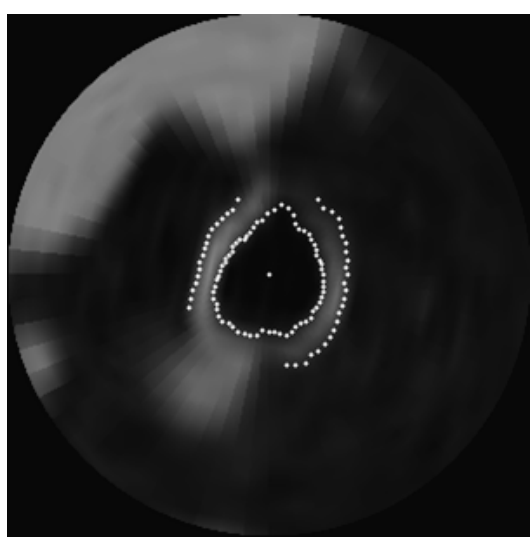

(c)

Figure 4. Exclusion of rays that belong to the attached structures. A radially reconstructed image of a cross section through an airway is shown. The radial rays (not shown) extend outward from the center point of the cross section. Inner and outer wall boundary locations for the rays are marked with white dots. (a) No exclusion (all rays). (b) Excluding the rays with thickness measurements greater than $3 / 4$ of the diameter estimate. (c) Further excluding the neighbors of eliminated rays.

vectors serve as the basis for the directions of the rays $R_{j}$. For $i=0$, we define $R_{x}(0)$ to be an arbitrarily oriented non-zero vector that is parallel to $P_{0} . R_{y}(0)$ can be obtained by taking cross product of $R_{x}(0)$ and $T_{0}$. For $i>0$, we calculate $R_{x}(i)$ as the projection of $R_{x}(i-1)$ to the current plane $P_{i}$. We first get a vector $R_{y}(i)$ that is mutually perpendicular to $R_{x}(i-1)$ and $T_{i}$ (Equation (4)), then find a vector that is mutually perpendicular to $R_{y}(i)$ and $T_{i}$, which becomes new $R_{x}(i)$ (Equation (5)).

$$
\begin{gathered}
R_{y}(i)=T_{i} \times R_{x}(i-1) . \\
R_{x}(i)=R_{y}(i) \times T_{i} .
\end{gathered}
$$
$(6))$.

Once we have $R_{x}$ and $R_{y}$, the set of evenly spaced rays $R_{j}$ is computed for each sampling plane $P_{i}$ (Equation

$$
R_{j}(i)=\frac{R_{x}(i)}{\left|R_{x}(i)\right|} \cos \alpha_{j}+\frac{R_{y}(i)}{\left|R_{y}(i)\right|} \sin \alpha_{j}, \quad \alpha_{j}=j 2 \pi / J, \quad j=0,1, \ldots, J-1 .
$$

Along each ray $R_{j}$, the intensities are sampled every $0.01 \mathrm{~mm}$ using trilinear interpolation (Equation (7)).

$$
I(i, j, k)=I\left(X_{i}+k(0.01) \frac{R_{j}(i)}{\left|R_{j}(i)\right|}\right), \quad k=0,1, \ldots, 1500
$$

The sampled intensities are stored in the form of a polygonal cylinder (Figure 3a) so that the rays $R_{j}$ go through the centers of triangles in the cylinder model. Our model provides a convenient framework that allows easy adjustment of both vertical and horizontal resolutions. Moreover, it allows for various analysis of the airway by mapping individual segment to a perfectly tubular structure.

\subsection{Airway Measurements}

Once we have the cylindrical representation of an airway segment, the actual measurement is taken from this model. For a given ray $R_{j}$, The Full width at half maximum (FWHM) principle is used to determine the inner and outer wall locations $i w\left(R_{j}\right)$ and $o w\left(R_{j}\right)$. The inner wall location is the boundary between the airway lumen and wall, and the outer wall location is the boundary between the airway wall and lung parenchyma. The boundary is determined to be the location where the intensity is at half way between the minimum and maximum values. The wall locations $i w$ and $o w$ are defined as the distance from the center point in a given layer to the boundaries. 
The lumen diameter (LD) of the airway is estimated by adding the inner wall locations of two opposite rays, as shown in Equation(8). The airway wall thickness (WT) is determined by the distance between the inner and outer wall locations (Equation (9)). For eacy airway segment, the average lumen diameter and wall thickness from all valid rays is reported.

$$
\begin{gathered}
L D=i w\left(R_{j}\right)+i w\left(R_{j+J / 2}\right) . \\
W T=o w\left(R_{j}\right)-i w\left(R_{j}\right) .
\end{gathered}
$$

For wall thickness measurement, not all rays can be used to get the outer wall locations since there may be attached vessel or other high intensity tissues next to the airway. We exclude the rays with wall thickness measurements greater than a threshold value from the wall thickness analysis. The threshold value for the wall thickness measurement was determined by multiplying $3 / 4$ to the lumen diameter estimate of the given segment. The rays that are near the excluded rays are also excluded since their wall thickness measurements may also be affected by the attached vessel or tissues. The effect of neighbor exclusion is visualized in Figure 4, where inner and outer wall boundaries are marked with white dots.

\section{EXPERIMENTAL DESIGN}

The automated method was evaluated on CT scans of 14 healthy subjects from a Weill Cornell database. All 14 cases were low dose $(120 \mathrm{kV}, 80 \mathrm{mAs})$, whole-lung scans with $1.25 \mathrm{~mm}$ thickness. Each subject had two scans that were taken within a 2 month interval and developed no symptoms in the interim; therefore we would expect no significant change in airway dimensions. The identical segments were visually located in the repeat scans, and the automated analysis was performed for each segment. The segments of segmental bronchi and beyond, where the entire airway segments are surrounded by lung parenchyma, were considered for this study.

The measurements were analyzed in two ways. First experiment considered the measurement changes in all segments from 14 subjects. For a patient with systemic airway disease, we would expect to see similar changes in all airway segments across the scan pair. To see how the measurement changed within each subject, we also performed per-patient analysis by considering overall change for each individual. For each patient, overall change in airway size was represented by the average change for all matched segments in that patient. For both analyses, average absolute change and $95 \%$ limits of agreement on measurement changes from scan 1 to scan 2 were computed.

\section{RESULTS}

Table 1. Limits of agreement for change in airway measurements.

\begin{tabular}{|l|c|c|c|c|}
\hline & \multicolumn{2}{|c|}{ Lumen diameter $(\mathrm{mm})$} & \multicolumn{2}{c|}{ Wall thickness (mm) } \\
\hline & All segments & Per-patient & All segments & Per-patient \\
\hline $95 \%$ limits of agreement & {$[-0.85,0.83]$} & {$[-0.57,0.47]$} & {$[-0.32,0.26]$} & {$[-0.16,0.10]$} \\
\hline
\end{tabular}

The total number of segments identified in both scans was 131. The average LD measurement for all identified segments was $4.44 \mathrm{~mm}$, and the average WT measurement was $1.80 \mathrm{~mm}$. Figure 5 shows a graph of the measurement change against the average of two measurements and shows that the measurement differences do not vary across airway sizes.

Table 1 shows the $95 \%$ limits of agreement on measurement changes for both all segments and per-patient analyses, and Figure 6 compares the average absolute measurement changes for the two analyses. For both LD and WT, per-patient analysis resulted in more consistent measurements across the scan pairs. For lumen diameter, average absolute measurement change for per-patient analysis was $0.19 \mathrm{~mm}$, compared to $0.31 \mathrm{~mm}$ when all segments were considered together. Likewise, for wall thickness, per-patient analysis resulted in average 


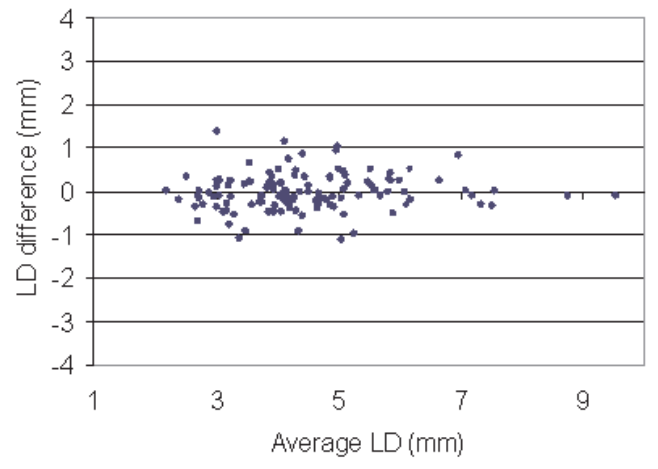

(a)

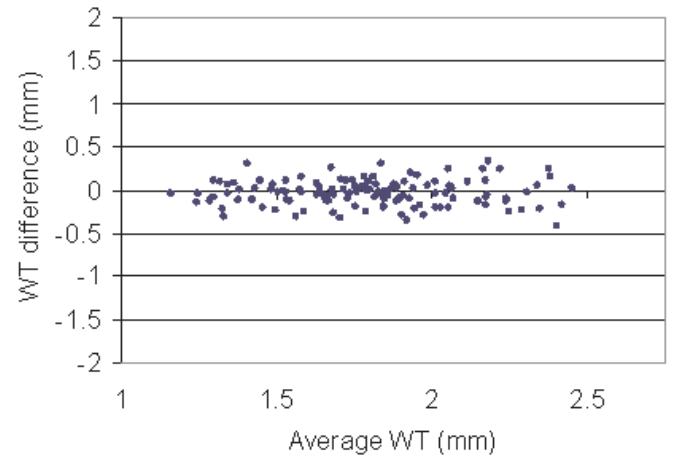

(b)

Figure 5. Bland-Altman plot of automated measurements. Measurement differences between two scans are plotted against average measurements. Each point represents an airway segment pair. (a) For lumen diameter measurements. (b) For wall thickness measurements.

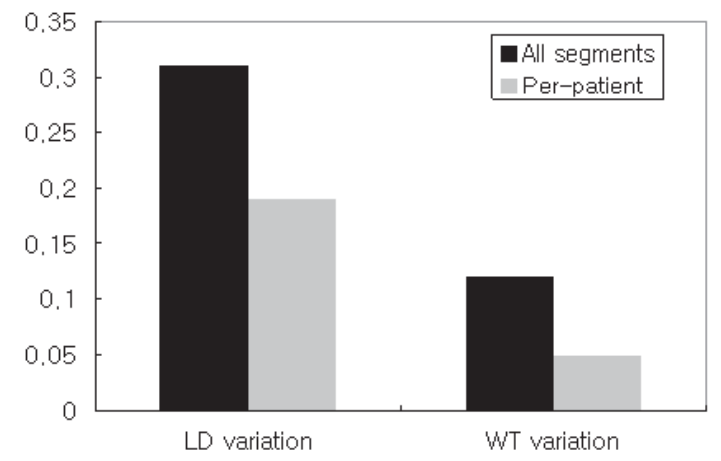

Figure 6. Comparison of airway measurement variation for considering all segments and individual patient. Average absolute changes in lumen diameter (LD) and wall thickness (WT) measurements are shown in mm. Variation is less when individual subject is analyzed separately. 
change of $0.05 \mathrm{~mm}$, compared to $0.12 \mathrm{~mm}$ for all segments. For all segments, the percentage variation for the automated measurements was $7.0 \%$ for $\mathrm{LD}$ and $6.7 \%$ for WT.

The results show that the per-patient analysis resulted in less measurement variation between the two scans than for the all segments analysis. The average absolute variation decreased by more than $35 \%$ for lumen diameter and more than $50 \%$ for wall thickness. The result is very encouraging since it means that with the automated measurements, we can monitor individual patients with a high degree of precision.

\section{CONCLUSION}

Accurate and robust measurements of human airway dimensions can be used to diagnose and monitor patients with respiratory diseases. In this paper, we propose an automated method that can measure lumen diameter and wall thickness of the airway segments. Our method is designed to work with arbitrarily oriented airway segments and require no manual intervention.

Our method has been evaluated with 131 segments from 14 repeat CT scan pairs. The results indicate that airway diameter and wall thickness can be measured with a high degree of precision, especially when measurements of all selected airway segments are averaged for a patient.

\section{REFERENCES}

[1] J. Reinhardt, N. D'Souza, and E. Hoffman, "Accurate measurement of intrathoracic airways," IEEE Transactions on Medical Imaging 16(6), pp. 820-827, 1997.

[2] Y. Nakano, J. Wong, P. de Jong, L. Buzatu, T. Nagao, H. Coxson, W. Elliott, J. Hogg, and P. Pare, "The Prediction of Small Airway Dimensions Using Computed Tomography," American Journal of Respiratory and Critical Care Medicine 171(2), p. 142, 2005.

[3] J. Tschirren, E. Hoffman, G. McLennan, and M. Sonka, "Intrathoracic airway trees: segmentation and airway morphology analysis from low-dose CT scans," IEEE Transactions on Medical Imaging 24(12), pp. 15291539, 2005.

[4] P. Berger, V. Perot, P. Desbarats, J. Tunon-de Lara, R. Marthan, and F. Laurent, "Airway wall thickness in cigarette smokers: Quantitative thin-section ct assessment," Radiology 235(3), pp. 1055-1064, 2005.

[5] K. Palágyi, J. Tschirren, and M. Sonka, "Quantitative analysis of three-dimensional tubular tree structures," Proc. SPIE Conf. Medical Imaging 5032, pp. 267-277, 2003.

[6] S. Wood, J. Hoford, E. Hoffman, E. Zerhouni, and W. Mitzner, "Quantitative 3D reconstruction of airway and pulmonary vascular trees using HRCT," Proceedings of SPIE 1905, pp. 316-323, 1993.

[7] G. Bertrand and G. Malandain, "A new characterization of three-dimensional simple points," Pattern Recognition Letters 15, pp. 169-175, 1994. 